\title{
The Role of Contrast Sensitivity in the Development of Binocular Vision: a Computational Study
}

\author{
Frankfurt Institute for Advanced Studies ${ }^{1}$, Frankfurt am Main, Germany \\ Hong Kong University of Science and Technology ${ }^{2}$, Hong Kong, China
}

Priamikov A. ${ }^{1}$, Narayan V. ${ }^{1}$, Shi B.E. ${ }^{2}$, and Triesch $J .^{1}$

The development of accurate binocular vision relies on the acquisition of disparity tuning and the calibration of vergence eye movements. Both processes are fundamentally limited by visual acuity, which gradually increases during the first year of life. It has been speculated that early limitations of visual acuity aid in the rapid learning of binocular disparity estimation [1], in analogy to Newport's "less-ismore" hypothesis [2]. This hypothesis states that a gradual increase in complexity of training signals allows the infant to efficiently acquire a skill, while unconstrained complexity in the training signals could be overwhelming. Here we assess the potential impact of early acuity limitations on the development of binocular vision in the context of the active efficient coding framework. We have recently proposed a computational model implemented on a physical robot that simultaneously learns to represent binocular disparity representations and to calibrate vergence eye movements [3]. In this active efficient coding approach, a generative model learns to encode sensory signals and a reinforcement learner learns to control eye movements with the objective of optimizing the encoding quality within the generative model (Fig. 1). The reinforcement learner receives an intrinsic reward signal that measures the encoding quality currently obtained by the generative model. This drives a self-calibrating learning of binocular disparity representations and vergence eye movements. To the best of our knowledge, this model is the first to explain how the development of disparity tuning and vergence eye movements mutually support each other. Here we extend this model to incorporate the development of visual acuity between birth and 8 months. To this end, we filter the visual input of the model according to measured contrast sensitivity functions at different ages [4,5]. Results of such filtering are shown in Figure 2. We find that the model does in fact learn faster, if visual acuity starts out poor and increases with time, supporting the less-is-more hypothesis. Furthermore, we find that the speed of acuity improvement needs to be "just right", i.e., neither too fast nor too slow for learning to be maximally efficient. Overall, our model thus suggests that early limitations of visual acuity might aid the learning agent in efficiently acquiring binocular vision skills.

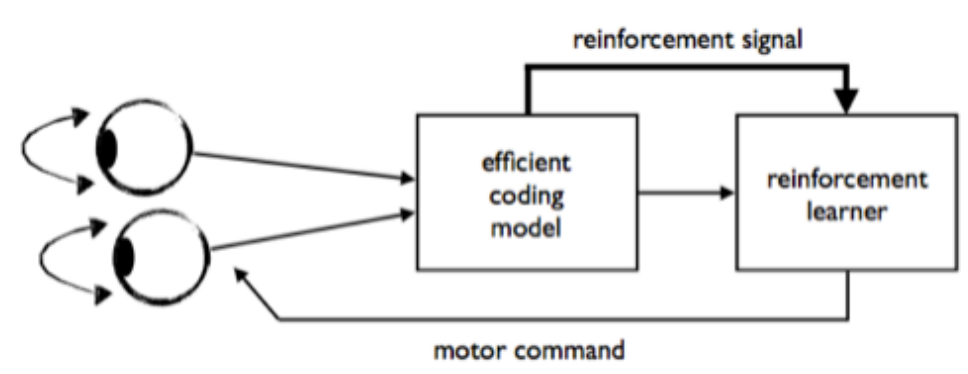

Figure 1. Active efficient coding framework.

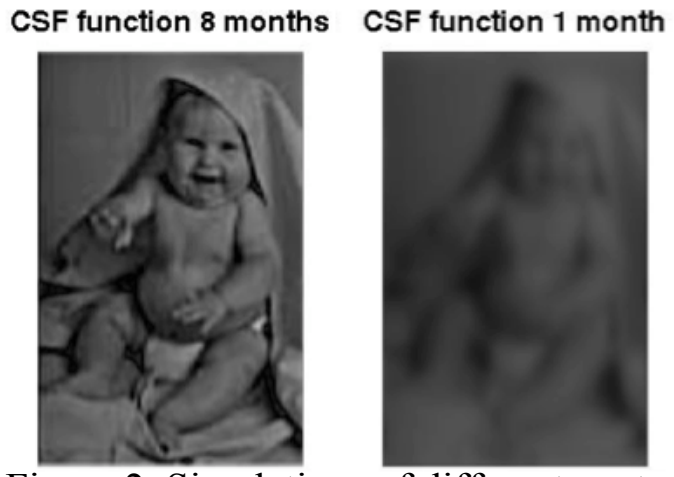

Figure 2. Simulations of different contrast sensitivity functions.

[1] Dominguez M. et al., Developmental constraints aid the acquisition of binocular disparity sensitivities, 2003.

[2] Newport E., Maturational constraints on language learning, 1990.

[3] Lonini L. et al., Autonomous learning of active multi-scale binocular vision, 2013.

[4] Teller D., Spatial and temporal aspects of infant color vision, 1998.

[5] Atkinson J. et al., Development of contrast sensitivity over the first 3 months of life in the human infant, 1977. 